\title{
Formal protocol for emergency treatment of ruptured intracranial aneurysms to reduce in-hospital rebleeding and improve clinical outcomes
}

\author{
Jaechan Park, MD, PhD, ${ }^{1,3}$ Hyunjin Woo, MD, ${ }^{1}$ Dong-Hun Kang, MD, ${ }^{1,2}$ Yong-Sun Kim, MD, PhD, ${ }^{2}$ \\ Min Young Kim, PhD, ${ }^{3}$ Im Hee Shin, PhD, ${ }^{4}$ and Sang Gyu Kwak, PhD ${ }^{4}$
}

Departments of ${ }^{1}$ Neurosurgery and ${ }^{2}$ Radiology, and ${ }^{3}$ Research Center for Neurosurgical Robotic Systems, Kyungpook National University, Daegu; and ${ }^{4}$ Department of Medical Statistics, School of Medicine, Catholic University of Daegu, Republic of Korea

\begin{abstract}
OBJECT While the incidence of a recurrent hemorrhage is highest within 24 hours of subarachnoid hemorrhage (SAH) and increases with the severity of the clinical grade, a recurrent hemorrhage can occur anytime after the initial SAH in patients with both good and poor clinical grades. Therefore, the authors adopted a 24-hour-a-day, formal protocol, emergency treatment strategy for patients with ruptured aneurysms to secure the aneurysms as early as possible. The incidences of in-hospital rebleeding and clinical outcomes were investigated and compared with those from previous years when broadly defined early treatment was used ( $<3$ days of $\mathrm{SAH})$.
\end{abstract}

METHODS During an 11-year period, a total of 1224 patients with a ruptured aneurysm were managed using a strategy of broadly defined early treatment between 2001 and 2004 (Period $B, n=423$ ), a mixture of early or emergency treatment between 2005 and 2007, and a formal emergency treatment protocol between 2008 and 2011 (Period $A, n=442$ ). Propensity score matching was used to adjust the differences in age, sex, modified Fisher grade, World Federation of Neurosurgical Societies (WFNS) clinical grade at admission, size and location of a ruptured aneurysm, treatment modality (clip placement vs coil embolization), and time interval from SAH to admission between the two time periods. The matched cases were allotted to Group A $(n=280)$ in Period A and Group B $(n=296)$ in Period B and then compared.

RESULTS During Period A under the formal emergency treatment protocol strategy, the catheter angiogram, endovascular coiling, and surgical clip placement were started at a median time from admission of 2.0 hours, 2.9 hours, and 3.1 hours, respectively. After propensity score matching, Group A showed a significantly reduced incidence of in-hospital rebleeding (2.1\% vs $7.4 \%, p=0.003)$ and a higher proportion of patients with a favorable clinical outcome (modified Rankin Scale score $0-3)$ at 1 month (87.9\% vs $79.7 \%$, respectively; $p=0.008)$. In particular, the patients with good WFNS grades in Group A experienced significantly less in-hospital rebleeding (1.7\% vs $5.7 \%$, respectively; $p=0.018$ ) and better clinical outcomes (1-month mRS score of $0-3: 93.8 \%$ vs $87.7 \%$, respectively; $p=0.021$ ) than the patients with good WFNS grades in Group B.

CONCLUSIONS Patients with ruptured aneurysms may benefit from a strategy of emergency application of surgical clip placement or endovascular coiling due to the reduced incidence of recurrent bleeding and improved clinical outcomes.

http://thejns.org/doi/abs/10.3171/2014.9.JNS131784

KEY WORDS emergency treatment; intracranial aneurysm; outcome; subarachnoid hemorrhage; vascular disorders; formal protocol

$\mathrm{W}$ HEN determining the management strategy for a ruptured intracranial aneurysm, the timing of the aneurysm obliteration by surgical clip placement or endovascular coiling remains somewhat controversial and varies according to individual institutions. Because aneurysmal rebleeding is a significant cause of morbidity and death in patients who survive the initial effects of the aneurysmal rupture, the general consensus on the optimum time for definitive treatment has shifted from late surgery (> 7 days after subarachnoid hemorrhage $[\mathrm{SAH}]$ ) to early

ABBREVIATIONS ACA = anterior cerebral artery; EACA = $\varepsilon$-aminocaproic acid; EVD = external ventricular drainage; ICA = internal carotid artery; IQR = interquartile range; $\mathrm{MCA}=$ middle cerebral artery; $\mathrm{mRS}=$ modified Rankin Scale; SAH = subarachnoid hemorrhage; VBA = vertebrobasilar artery; WFNS = World Federation of Neurosurgical Societies.

ACCOMPANYING EDITORIAL See pp 381-382. DOI: 10.3171/2014.8.JNS141613.

SUBMITTED August 21, 2013. ACCEPTED September 18, 2014.

INCLUDE WHEN CITING Published online November 18, 2014; DOI: 10.3171/2014.9.JNS131784.

DISCLOSURE This study was supported by a grant (no. A100870) from the Korea Healthcare Technology R \& D Project, Ministry of Health \& Welfare, Republic of Korea. 
surgery ( $<3$ days after $\mathrm{SAH}$ ), based on the results of a previous cooperative study. ${ }^{4,10}$ Thus, most institutions aim to treat aneurysms within 24-72 hours, with definitive treatment undertaken during standard operating hours when the conditions of the neurovascular team are optimized (i.e., during times when team members are available and not fatigued) ${ }^{6}$ Furthermore, because the incidence of rebleeding is greatest within the first 24 hours of $\mathrm{SAH}, 3,9$ ultra-early treatment (within 24 hours) of SAH has recently been introduced and adopted as a goal at many institutions.

Moreover, a recurrent hemorrhage can occur anytime after the initial SAH in patients with good and poor clinical grades, in which the incidence is highest within 24 hours of SAH and increases with the severity of the clinical grade. Therefore, the present authors, including neurovascular surgeons and neurointerventionists, adopted a 24-hour-a-day, formal emergency treatment protocol strategy for patients with ruptured aneurysms to secure the aneurysms as early as possible. The timing of the aneurysm treatment was not affected by the rupture time, admission time, clinical grade, or age of the patient. Accordingly, this study investigates the changes in the incidence of in-hospital rebleeding and clinical outcomes after adopting the formal emergency treatment protocol strategy.

\section{Methods \\ Patient Population}

During an 11-year period, a total of 1224 patients with an aneurysmal SAH were managed at the present tertiary referral center (Kyungpook National University Hospital). Between 2001 and 2004, a strategy of broadly defined early treatment $(<3$ days of SAH) was applied, in which catheter angiography was performed on Day 1 after admission, followed by surgical clip placement or endovascular coiling on Day 2 after admission. In the case of intracranial hypertension due to a large intracerebral hematoma or subdural hematoma, the patient was treated with an emergency craniotomy and aneurysm clipping, while acute hydrocephalus was managed by bedside placement of external ventricular drainage (EVD).

Between 2005 and 2007, an early or emergency treatment strategy was applied according to the neurosurgeon in charge. However, from 2008, a formal emergency treatment protocol strategy was strictly applied. Medical records were reviewed to obtain relevant clinical information, and all radiological data in this study were obtained using an electronic picture archiving and communication system.

An SAH diagnosis was based on the results of CT or occasionally spinal tapping, while rebleeding was defined as the recurrence of a headache or occurrence of mental change with a sudden onset after the initial SAH, accompanied by corresponding CT findings. Cases of non-giant saccular aneurysms were included, whereas cases of SAH caused by arterial dissection, a perimesencephalic venous hemorrhage, arteriovenous malformation, or unknown etiology were excluded. The clinical grade was determined at admission using a World Federation of Neurosurgical Societies (WFNS) grade, while the time at which the SAH occurred was determined based on the medical records, including an interview with the patient and his or her family. When the time of the SAH was unknown, these cases (39 patients) were excluded. All patients received standard neurocritical care, including adequate hydration, nutritional support, nimodipine, and neurological monitoring. However, no antifibrinolytic agent, such as $\varepsilon$-aminocaproic acid (EACA), was administered.

The demographic and clinical characteristics, in-hospital rebleeding, and treatment outcomes for the patients from 2008 to 2011 (Period A, $\mathrm{n}=442$ ) and from 2001 to 2004 (Period $B, n=423$ ) were analyzed, whereas the patient data from 2005 to 2007 (transitional period) were excluded. The study was reviewed and approved by an institutional ethics committee.

\section{Formal Emergency Treatment Protocol of Ruptured Aneurysms}

The management protocol for a ruptured aneurysm during Period A (2008-2011) is presented in Fig. 1. The protocol was strictly applied in an emergency manner without delay. When a patient presented with a severe headache or mental deterioration with a sudden onset, an SAH diagnosis was primarily obtained on the basis of an immediate CT scan.

Within 1 hour of the SAH diagnosis, catheter angiography was performed to determine the cause of the SAH and investigate the feasibility of endovascular coiling. The final treatment decision (surgical vs endovascular) was made jointly on a case-by-case basis by the neurovascular surgeon (J.P.) and endovascular interventionist (Y.S.K. or D.H.K.) in the angiography suite. The surgical clip placement or endovascular coiling was then performed immediately after obtaining consent from the patient's family.

When the initial CT scan strongly indicated surgical treatment, as in the case of an associated large intracerebral hemorrhage or subdural hemorrhage, CT angiography was performed instead of catheter angiography to save time and facilitate a craniotomy.

As described in a previous report by the current authors, surgical treatment was favored over endovascular treatment for patients with the following angiographic or clinical findings: 1) high tortuosity of the proximal vessels; 2) difficult navigation of the microcatheter into the aneurysm;2,20 3 ) very small aneurysms $(<3 \mathrm{~mm}$ in diameter); ${ }^{2,8,20} 4$ ) large and giant aneurysms, ${ }^{21,29} 5$ ) wide-necked aneurysms necessitating stent- or balloon-assisted technology; ${ }^{13,21} 6$ ) aneurysms with an arterial branch incorporated into the sac; ;1,19 7) a fusiform or complex aneurysm configuration; ${ }^{2,28} 8$ ) aneurysms with a basal outpouching or stalklike narrow neck suggesting the possibility of a basal rupture;22,24 9) aneurysms with an intraluminal thrombus; ${ }^{17} 10$ ) blood blister-like aneurysms of the internal carotid artery (ICA); ${ }^{1,26}$ 11) aneurysms associated with a large intracerebral hemorrhage or subdural hemorrhage; or 12) aneurysms compressing adjacent cranial nerves, ${ }^{23}$ as long as the patients had no problems related to comorbidity or surgical accessibility.

\section{Statistical Analysis}

Propensity score matching was used to match the vari- 


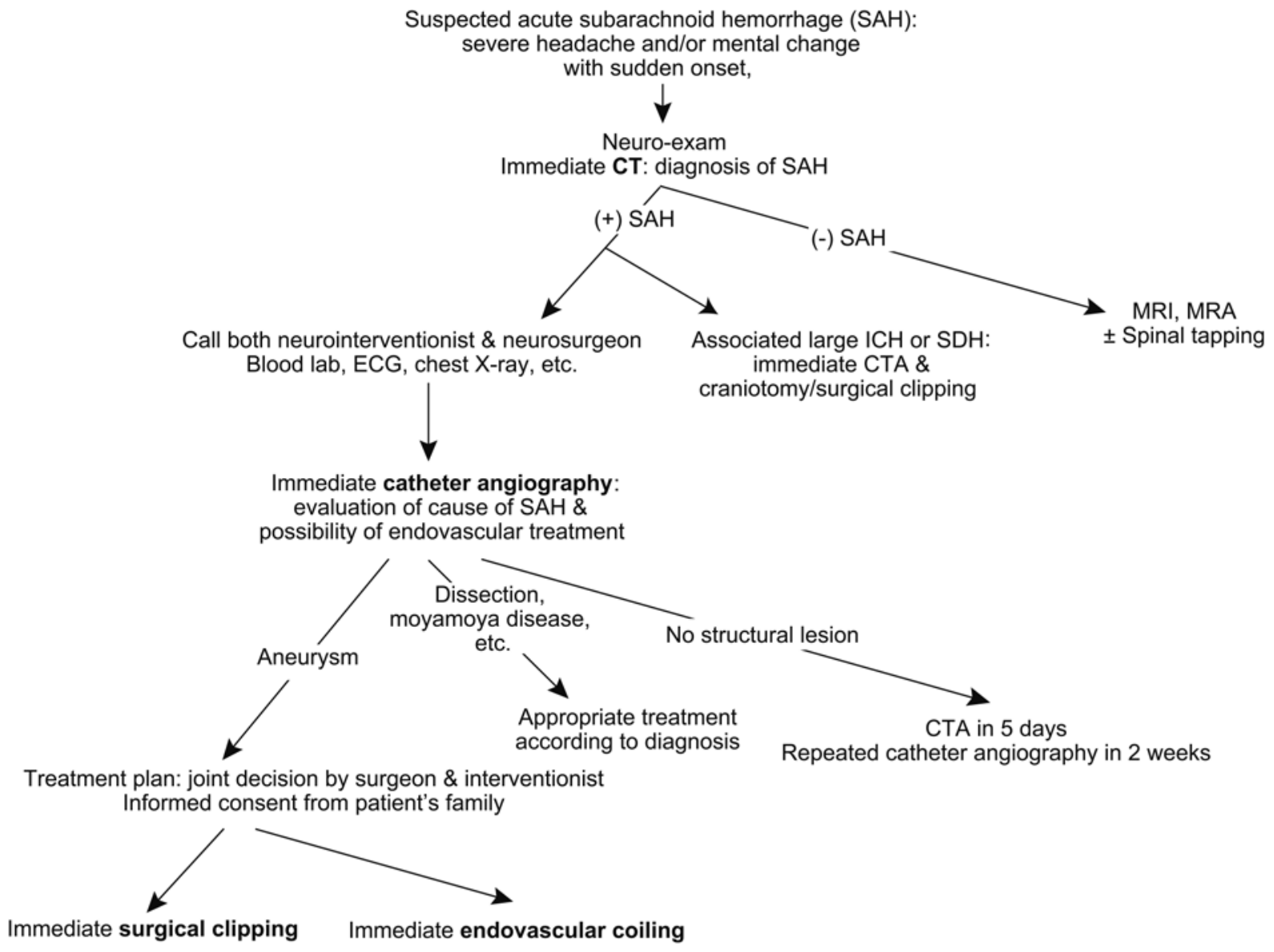

FIG. 1. Current formal emergency treatment protocol of SAH. CTA = CT angiography; $E C G=$ electrocardiography; $I C H=$ intracerebral hematoma; MRA = MR angiography; SDH = subdural hematoma.

ables of age, sex, WFNS clinical grade (1-3 vs 4-5) on admission, modified Fisher grade, location and maximum diameter of the ruptured aneurysm, time interval from SAH to admission, and treatment modality (clip placement vs coil embolization) between the two treatment groups. The logit used to perform this was a caliper, in which the maximum width of the caliper was the confounding variable for the standard deviation of the logit for the matching variable. In addition, a fixed seed number was used to maintain the same result for the matching data set. First, the propensity score matching was separately applied to the patients with rebleeding (caliper = logit, caliper size 0.145 , ratio $=4: 1$, seed number $=34567$ ) and those without rebleeding (caliper $=$ logit, caliper size 0.06 , ratio $=1: 1$, seed number $=34567)$ as shown in Fig. 2. The matched cases were then grouped in Period A or Period B according to the time of admission and treatment strategy.

For the qualitative variables, the results were described based on the frequency using a percentage, and the comparisons between Period A and Period B were performed using a chi-square test. For quantitative variables, the results were described as means \pm standard deviations, and the comparisons between Period A and Period B were performed using a 2-sample t-test. Subgroup analyses were also performed for the patients with good and poor WFNS grades. All the tests were 2-sided, and a p value of less than
0.05 was considered statistically significant. The statistical analyses were performed using SPSS (version 19.0, SPSS Inc.) and $\mathrm{R}$ (version 2.15, Lucent Technologies Inc.).

\section{Results}

\section{Baseline Patient Characteristics Before Propensity Score Matching}

The baseline data, including the clinical characteristics, treatment modalities, time interval to treatment, and in-hospital rebleeding, are summarized in Table 1. A total of 442 and 423 patients with a ruptured aneurysm were treated during Periods A and B, respectively. For Period A, the mean age of the patients was $55.7 \pm 12.9$ years, and 293 patients $(66.3 \%)$ were female. The WFNS clinical grade at admission was $1-3$ for 374 patients $(84.6 \%)$ and 4 or 5 for 68 patients (15.4\%), while the modified Fisher grade was 1 or 2 for 261 patients $(59.0 \%)$. The ruptured aneurysms were measured based on the maximum diameter (mean $6.9 \pm 4.4$ $\mathrm{mm})$ and located in the ICA $(\mathrm{n}=132,29.9 \%)$, anterior cerebral artery $(\mathrm{ACA} ; \mathrm{n}=165,37.3 \%)$, middle cerebral artery (MCA; $n=118,26.7 \%)$, and vertebrobasilar artery (VBA; $\mathrm{n}=27,6.1 \%)$. Surgical clip placement as the treatment modality for obliterating the ruptured aneurysm was applied to 275 patients in the Period A group (62.2\%), while endovascular coiling was applied to 167 patients (37.8\%). 


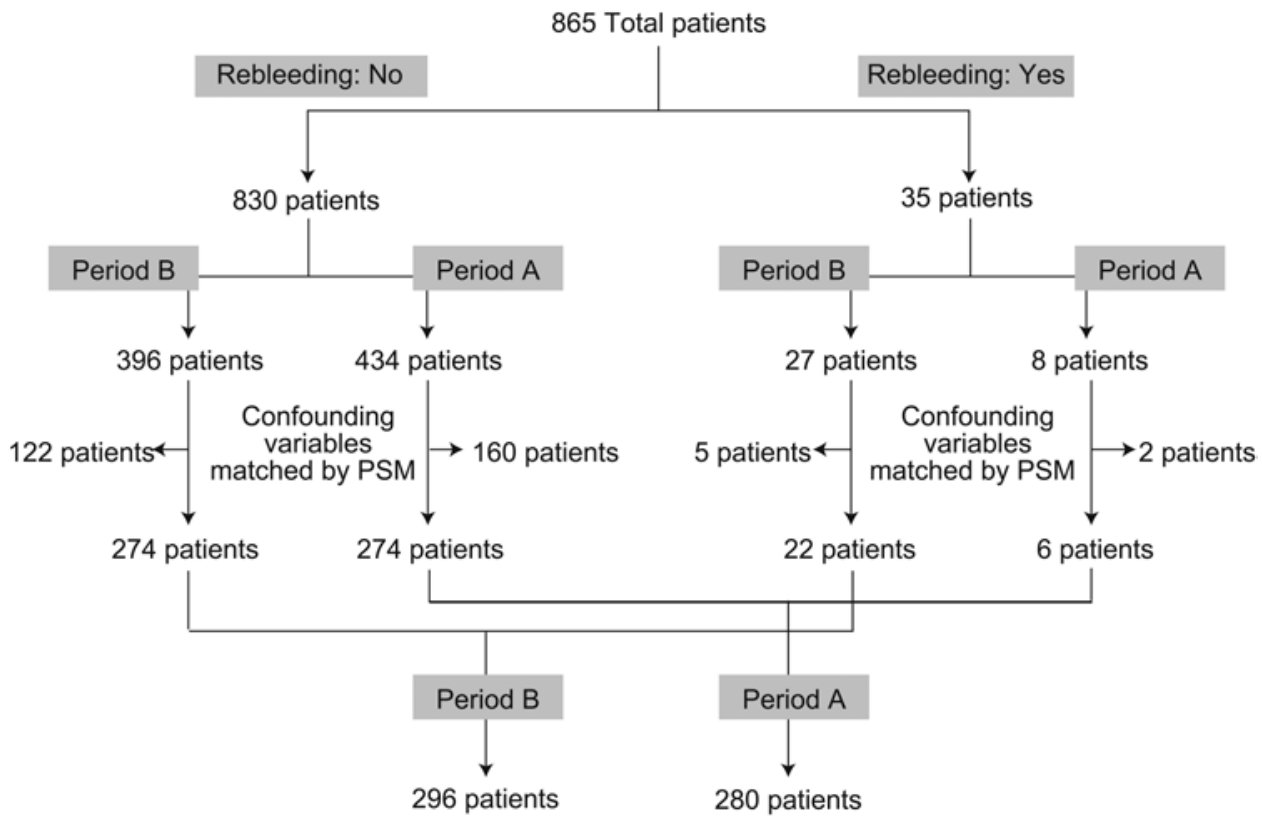

FIG. 2. Propensity score matching to adjust differences in age, sex, WFNS grade, modified Fisher grade, location and maximum diameter of the ruptured aneurysm, time interval from SAH to admission, and treatment modality. PSM = propensity score matching.

While there was no significant difference in the age, sex, WFNS clinical grade, site of the ruptured aneurysm, or time interval from SAH to admission between the patients from Period A and the patients from Period B, there was a difference in the modified Fisher grade, size of the ruptured aneurysm, and applied treatment modality.

\section{Time to Treatment and In-Hospital Rebleeding}

During Period A, surgical treatment was started at a median (interquartile range [IQR]) time from admission of 3.1 (IQR 1.6) hours, while endovascular coiling was started at a median time from admission of 2.9 (IQR 2.2) hours. In the case of 413 patients (93.4\%), definitive treatment to obliterate the aneurysm was started within 6 hours after admission. The median time from SAH to definitive treatment was 7.5 (IQR 10.0) hours for surgical clipping and 7.9 (IQR 11.5) hours for endovascular coiling.

Meanwhile, during Period B, surgical treatment was started at a median (IQR) time from admission of 39.7 (IQR 43.3) hours, while endovascular coiling was started at a median time from admission of 49.4 (IQR 97.2) hours.

During Period A, a formal emergency treatment protocol strategy was strictly applied, regardless of the hour (both day and night). For the 146 patients (33.0\%) admitted during the night (7 p.m. to 7 a.m.), the catheter angiography, endovascular treatment, and surgical treatment were started at a median (IQR) time from admission of 1.8 (IQR 1.3) hours, 2.4 (IQR 1.6) hours, and 3.1 (IQR 1.6) hours, respectively. These time intervals were not significantly different from those of the 296 patients (67.0\%) admitted during the day (7 a.m. to 7 p.m.), at 2.0 (IQR 1.2) hours, 3.0 (IQR 1.9) hours, and 3.3 (IQR 1.8) hours, respectively.

The incidence of in-hospital rebleeding before definitive treatment was 6.4\% (27/423 patients) during Period B and only $1.8 \%$ (8/442 patients) during Period A (Table 2).
During Period B, 63.0\% (17/27 patients) of the rebleeding cases occurred within 24 hours after admission, representing $4.0 \%$ (17/423 patients) of the total admitted patients, and immediate rebleeding ( $<4$ hours) after admission occurred in 6 patients $(6 / 423,1.4 \%)$. During Period A, while the incidence of immediate rebleeding was not reduced at $1.8 \%$ ( $8 / 442$ patients), there were no recurrent hemorrhages after 4 hours postadmission. For the patients who experienced immediate rebleeding, this occurred at a median time from SAH of 3.5 (IQR 2.2) hours and 3.5 (IQR 2.4) hours during Period A and B, respectively (Table 2).

Procedure-related rebleeding was also observed in the current series. In 4 of the 27 rebleeding cases during Period B, a recurrent hemorrhage occurred during a bedside ventriculostomy for acute hydrocephalus. In 1 of the 8 rebleeding cases during Period $\mathrm{A}$, a recurrent hemorrhage occurred during catheter angiography performed 2.8 hours after SAH.

A total of 35 patients developed in-hospital rebleeding during Periods A and B. Twenty-one (60\%) of these patients presented with WFNS Grade 1,2, or 3, representing $2.9 \%$ of the 728 patients with good WFNS grades, while the remaining $14(40 \%)$ patients were admitted with WFNS Grade 4 or 5 , representing $10.2 \%$ of the 137 patients with poor WFNS grades.

\section{Comparison of In-Hospital Rebleeding and Clinical Outcomes After Propensity Score Matching}

To adjust the differences in age, sex, WFNS grade at admission, modified Fisher grade, aneurysm size and location, treatment modality, and time interval from SAH to admission between Periods A and B, all patients were assigned a propensity score and then matched based on that score. The results were a closely paired group of 576 patients with small differences between the groups (Table 3). After propensity score matching, the data for Group A 
TABLE 1. Clinical characteristics and incidence of in-hospital rebleeding according to different strategies of treatment timing in 865 patients with ruptured aneurysms

\begin{tabular}{|c|c|c|c|}
\hline Variable & Period $A^{*}$ & Period $\mathrm{B}^{*}$ & p Value \\
\hline Total patients & 442 & 423 & \\
\hline Age (yrs) & & & $0.764 \S$ \\
\hline Mean \pm SD & $55.7 \pm 12.9$ & $55.5 \pm 11.6$ & \\
\hline Median (IQR) & $55.0(21.0)$ & $55.0(17.0)$ & \\
\hline Females (\%) & $293(66.3)$ & $298(70.4)$ & $0.189 \ddagger$ \\
\hline WFNS Grade 4 or $5(\%)$ & $68(15.4)$ & $69(16.3)$ & $0.709 \ddagger$ \\
\hline Modified Fisher Grade 3 or 4 (\%) & $181(41.0)$ & $257(60.8)$ & $0.000 \S$ \\
\hline Aneurysm size (mm) & & & $0.000 \S$ \\
\hline Mean \pm SD & $6.9 \pm 4.4$ & $5.8 \pm 2.7$ & \\
\hline Median (IQR) & $6.0(4.0)$ & $5.3(3.2)$ & \\
\hline Aneurysm site (\%) & & & $0.062 \ddagger$ \\
\hline ICA & $132(29.9)$ & $123(29.1)$ & \\
\hline ACA & $165(37.3)$ & $172(40.7)$ & \\
\hline MCA & $118(26.7)$ & $91(21.5)$ & \\
\hline VBA & $27(6.1)$ & $32(7.6)$ & \\
\hline Unknown & $0(0)$ & $5(1.2)$ & \\
\hline Treatment (\%) & & & $<0.001 \ddagger$ \\
\hline Clip placement & $275(62.2)$ & $322(76.1)$ & \\
\hline Coil embolization & $167(37.8)$ & $92(21.7)$ & \\
\hline None & $0(0)$ & $9(2.1)$ & \\
\hline \multicolumn{4}{|l|}{ Time interval (hrs) } \\
\hline SAH to admission & & & $0.093 \S$ \\
\hline Mean \pm SD & $17.6 \pm 39.0$ & $13.4 \pm 33.4$ & \\
\hline Median (IQR) & $3.8(9.5)$ & $3.3(6.0)$ & \\
\hline Admission to DS angiography & & & $<0.001 \S$ \\
\hline Mean \pm SD & $2.2 \pm 1.2$ & $22.6 \pm 29.1$ & \\
\hline Median (IQR) & $2.0(1.0)$ & $18.0(16.0)$ & \\
\hline Admission to clip placement & & & $<0.001 \S$ \\
\hline Mean \pm SD & $3.5 \pm 1.5$ & $56.8 \pm 60.2$ & \\
\hline Median (IQR) & $3.1(1.6)$ & $39.7(43.3)$ & \\
\hline Admission to coil embolization & & & $<0.001 \S$ \\
\hline Mean \pm SD & $3.2 \pm 1.8$ & $86.7 \pm 89.3$ & \\
\hline Median (IQR) & $2.9(2.2)$ & $49.4(97.2)$ & \\
\hline In-hospital rebleed (\%) & $8(1.8)$ & $27(6.4)$ & $0.001 \ddagger$ \\
\hline \multicolumn{4}{|l|}{ Time to rebleed (hrs) } \\
\hline Admission to rebleed & & & $<0.001 \S$ \\
\hline Mean \pm SD & $2.2 \pm 1$ & $21.5 \pm 22.1$ & \\
\hline Median (IQR) & $2.0(1.3)$ & $11.4(36.0)$ & \\
\hline SAH to rebleed & & & $0.732 \S$ \\
\hline Mean \pm SD & $27.9 \pm 48.6$ & $37.5 \pm 73.6$ & \\
\hline Median (IQR) & $3.7(50.2)$ & $16.4(38.0)$ & \\
\hline \multicolumn{4}{|c|}{$\begin{array}{l}\text { DS = digital subtraction. } \\
\text { * Period } A=\text { period with formal emergency treatment between } 2008 \text { and } 2011 \text {; Period } B=\text { period with broadly defined early treatment }(<3 \text { days } \\
\text { of SAH) between } 2001 \text { and } 2004 \text {. } \\
\dagger \text { Values in bold are statistically significant. } \\
\ddagger \text { Chi-square test. } \\
\S \text { Two-sample t-test. }\end{array}$} \\
\hline
\end{tabular}


TABLE 2. Incidence of in-hospital rebleeding according to time after admission in patients with a ruptured aneurysm

\begin{tabular}{ccccc}
\hline Period $(\mathrm{yrs})^{*}$ & $\begin{array}{c}\text { Time From Admission to } \\
\text { Rebleed (hrs) }\end{array}$ & No. of Patients (\%) & $\begin{array}{c}\text { Mean Time From SAH } \\
\text { to Rebleed } \pm \text { SD (hrs) }\end{array}$ & $\begin{array}{c}\text { Median Time From SAH } \\
\text { to Rebleed (IQR) }\end{array}$ \\
\hline A (2008-2011) & $0 \leq \mathrm{AR}<4$ & $8(1.8)$ & $12.8 \pm 24.8$ & $3.5(2.2)$ \\
\hline & $4 \leq \mathrm{AR}<8$ & 0 & & \\
\hline $8 \leq \mathrm{AR}<24$ & 0 & & \\
\hline $\mathrm{AR} \geq 24$ & 0 & & $3.5(2.4)$ \\
\hline Total & $8(1.8)$ & $3.2 \pm 1.3$ & $10.4(6.2)$ \\
\hline B (2001-2004) & $6(1.4)$ & $9.8 \pm 3.4$ & $17.7(7.2)$ \\
\hline & $4 \leq \mathrm{AR}<8$ & $5(1.2)$ & $18.3 \pm 5.3$ & $47.5(33.2)$ \\
\hline & $6 \leq \mathrm{AR}<24$ & $6(1.4)$ & & \\
\hline
\end{tabular}

$\mathrm{AR}=$ time between admission and rebleed.

* There were 442 total patients in Period A, and 423 patients in Period B.

$(\mathrm{n}=280)$ during Period A were compared with the data for Group B $(\mathrm{n}=296)$ during Period B.

The incidence of in-hospital rebleeding was significantly lower at 2.1\% (6/280 patients) for Group A when compared with Group B at 7.4\% (22/296 patients; $\mathrm{p}=$ 0.003 ; Table 3). The proportion of patients with a favorable clinical outcome (mRS score $0-3$ ) at 1 month after admission was significantly higher for Group A (87.9\%, 246/280

TABLE 3. Clinical characteristics, incidence of in-hospital rebleeding, and treatment outcomes in patients with a ruptured aneurysm after propensity score matching

\begin{tabular}{|c|c|c|c|}
\hline Characteristic & Group A (2008-2011) & Group B (2001-2004) & p Value* \\
\hline Total patients & 280 & 296 & \\
\hline Mean age $\pm S D$ (yrs) & $55.8 \pm 12.8$ & $55.5 \pm 11.7$ & $0.785 \ddagger$ \\
\hline Female (\%) & $185(66.1)$ & $213(72)$ & $0.126 \dagger$ \\
\hline WFNS Grade 4 or $5(\%)$ & $30(10.7)$ & $52(17.6)$ & $0.231 \dagger$ \\
\hline Modified Fisher Grade 3 or 4 (\%) & $132(47.1)$ & $153(51.7)$ & $0.603 \ddagger$ \\
\hline Mean aneurysm size $\pm \mathrm{SD}(\mathrm{mm})$ & $6.8 \pm 3.8$ & $6.2 \pm 2.7$ & $0.025 \ddagger$ \\
\hline Aneurysm site (\%) & & & $0.151 \dagger$ \\
\hline ICA & $84(30)$ & $90(30.4)$ & \\
\hline ACA & $100(35.7)$ & $116(39.2)$ & \\
\hline MCA & $78(27.9)$ & $64(21.6)$ & \\
\hline VBA & $18(6.4)$ & $22(7.4)$ & \\
\hline Unknown & $0(0)$ & $4(1.4)$ & \\
\hline Treatment (\%) & & & $0.009 \dagger$ \\
\hline Clip placement & $200(71.4)$ & $224(75.7)$ & \\
\hline Coil embolization & $80(28.6)$ & $65(22)$ & \\
\hline None & $0(0)$ & $7(2.4)$ & \\
\hline \multicolumn{4}{|l|}{ Time interval in hrs (mean \pm SD) } \\
\hline SAH to admission & $17.1 \pm 37.6$ & $15 \pm 37$ & $0.510 \ddagger$ \\
\hline Admission to DS angiography & $2.1 \pm 1.2$ & $24.6 \pm 32.5$ & $0.000 \ddagger$ \\
\hline Admission to clip placement & $3.6 \pm 1.7$ & $58.4 \pm 62.3$ & $0.000 \ddagger$ \\
\hline Admission to coil embolization & $3.4 \pm 2.1$ & $91.7 \pm 98.5$ & $0.000 \ddagger$ \\
\hline In-hospital rebleed (\%) & $6(2.1)$ & $22(7.4)$ & $0.003 \dagger$ \\
\hline \multicolumn{4}{|l|}{ Time to rebleed in hrs (mean \pm SD) } \\
\hline Admission to rebleed & $1.9 \pm 0.9$ & $23.3 \pm 23.3$ & $0.000 \ddagger$ \\
\hline SAH to rebleed & $3.4 \pm 0.9$ & $41.5 \pm 81.1$ & $0.267 \ddagger$ \\
\hline 1-month mRS score 4-6 (\%) & $34(12.1)$ & $60(20.3)$ & $0.008 \dagger$ \\
\hline
\end{tabular}

* Values in bold are statistically significant.

$\dagger$ Chi-square test.

$\ddagger$ Two sample t-test. 
patients) when compared with Group B (79.7\%, 236/296 patients; $\mathrm{p}=0.008)$.

\section{WFNS Grade-Based Subgroup Analysis of Matching Data}

After propensity score matching, the patients were grouped according to their WFNS grade at admission as follows: those with good WFNS grades (1-3), and those with poor WFNS grades (4 or 5). A subgroup analysisincluding those patients with good WFNS grades in Group A versus Group B, and those with poor WFNS grades in Group A versus Group B-then showed closely paired groups without any significant difference for all the critical data, including age, sex, modified Fisher grade, size and site of the ruptured aneurysm, treatment modality, and time interval from SAH to admission (Table 4).

When comparing the patients with good WFNS grades who received emergency treatment (Group A) with those who received early treatment (Group B), patients in Group A experienced significantly lower in-hospital rebleeding ( $1.7 \%$ vs $5.7 \%$, respectively; $\mathrm{p}=0.018)$ and better clinical outcomes (1-month mRS score $0-3: 93.8 \%$ vs $87.7 \%$, respectively; $\mathrm{p}=0.021)$. However, when comparing the patients with poor WFNS grades (Group A vs B), the differences in the rebleeding incidence and clinical outcomes did not reach statistical significance.

\section{Discussion}

A previous international cooperative study on the timing of aneurysm surgery reported a rebleeding incidence of 5.7\% among patients in the early surgery group $(<3$ days of SAH). ${ }^{9} 10$ Specifically, the rebleeding incidence within the first 24 hours of SAH was $4.1 \%$. These incidences are comparable to those for the early treatment patients (Pe$\operatorname{riod} \mathrm{B}$ ) in the current study: $6.4 \%$ overall incidence, and $4.0 \%$ within 24 hours of SAH.

According to the present data, the strategic change from early treatment to the formal emergency treatment protocol achieved an approximately 5\% absolute risk reduction of in-hospital rebleeding. In particular, an absolute risk reduction of approximately $2 \%$ was achieved for recurrent hemorrhages within 24 hours after admission. This effect of treatment timing on the incidence of recurrent hemorrhages before definitive treatment will surely be more sig-

TABLE 4. Comparison of clinical characteristics, incidence of in-hospital rebleeding, and treatment outcomes of good and poor WFNS grade patients in Groups A and B after propensity score matching

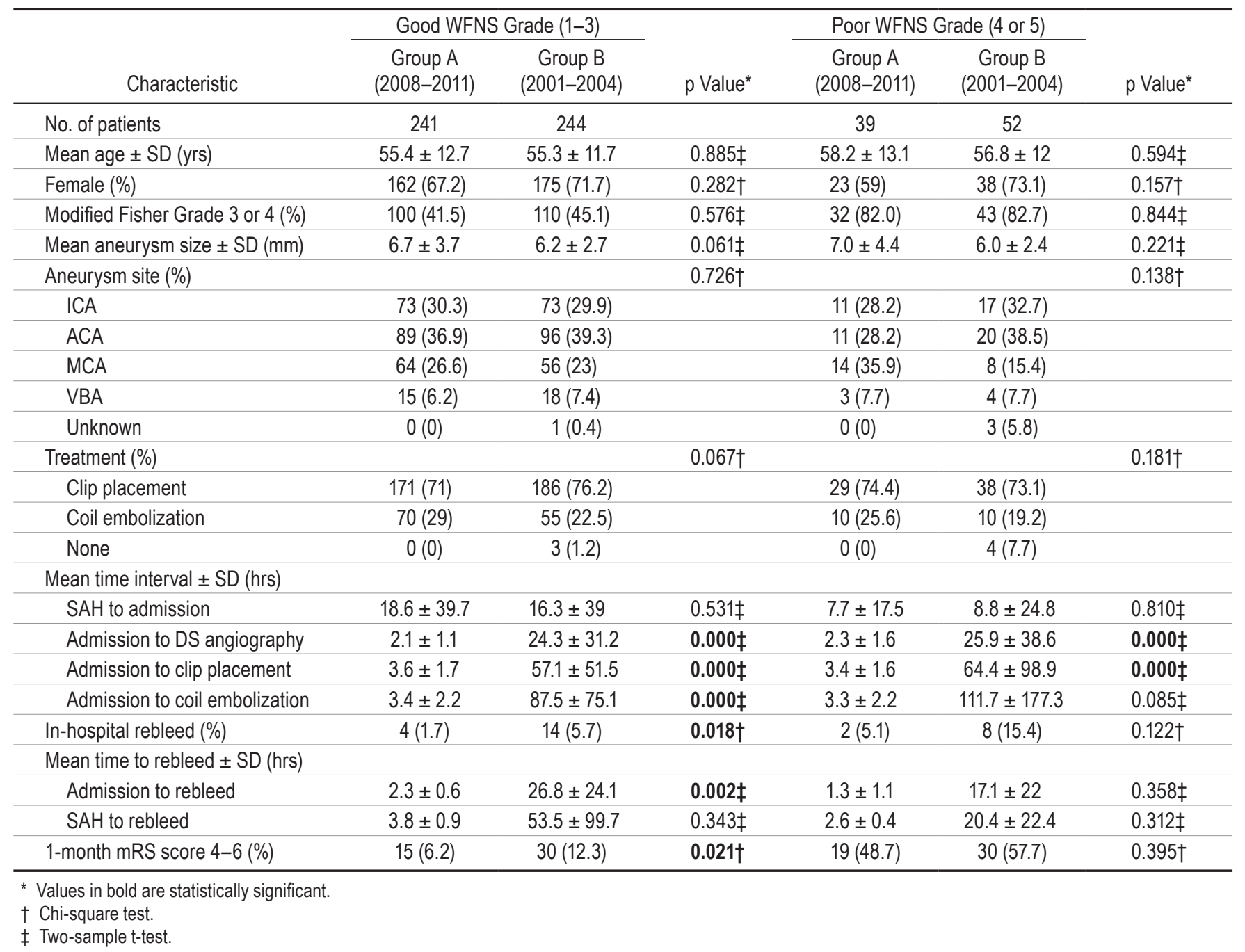


nificant in primary neurosurgical referral hospitals, where most patients are referred directly from emergency department physicians at the time of acute presentation..$^{16}$

Although the incidence of rebleeding increases with the severity of the clinical grade, ${ }^{5,15}$ rebleeding is more troublesome for patients with good WFNS grades. In the present series, the majority of patients had good WFNS grades $(84 \%)$. Moreover, rebleeding in patients with good WFNS grades invariably leads to significant neurological and clinical deterioration.

When using the formal emergency treatment protocol, the time during which the ruptured aneurysm remained unsecured was maximally reduced, while the need for bedside EVD was also minimized. Instead, the bedside EVD was replaced by an intraoperative ventriculostomy after dural opening during the craniotomy, which reduces the risk of recurrent hemorrhage. ${ }^{12}$ In the current series of inhospital rebleeding, 4 (14.8\%) of the 27 patients developed a recurrent hemorrhage during bedside EVD in Period B.

Cerebral angiography that is performed immediately after SAH can carry an increased risk of recurrent hemorrhage. Kusumi et al. ${ }^{14}$ reported a $23.9 \%$ incidence of recurrent hemorrhage during angiography when it was performed within 3 hours of SAH and recommended its avoidance. The in-hospital rebleeding in the current case series also included a case of recurrent hemorrhage during angiography within 3 hours of SAH. Thus, careful angiography, avoiding a contrast agent power injector, and appropriate medical management of hypertension are recommended.

Notwithstanding, the propensity score matching data from the current study revealed that Group A, which received emergency treatment for a ruptured aneurysm, experienced better clinical outcomes when compared with Group B, which received early treatment. This was particularly evident among the patients with good WFNS grades and was associated with a substantial reduction of in-hospital rebleeding. However, it should be noted that the better clinical outcomes may also have been affected by certain unevaluated factors, such as increased clinical experience, improved endovascular devices, and better organized multidisciplinary treatment.

To reduce recurrent hemorrhage, many neurovascular surgeons already endeavor to apply urgent treatment for a ruptured aneurysm within 24 hours after admission. Furthermore, there is no reason not to apply a strategy of emergency treatment for ruptured aneurysms if the neurovascular surgeons, interventionists, and anesthesiologists are all experienced and well organized as a team to apply emergency treatment. Yet, there remains stiff opposition to adopting a strategy of emergency treatment, mainly from doctors and institutions. An increased burden is placed on a small number of neurovascular surgeons and interventionists. Especially at night, the procedures can be performed under suboptimal conditions, when the surgical team may not be ideal and some members may be fatigued. ${ }^{6}$ The costs are also increased to provide coverage 24 hours a day, 7 days a week.

The critical benefit of using the emergency treatment strategy, instead of urgent treatment within 24 hours after admission, is to prevent recurrent hemorrhage in 2 out of 100 patients with a ruptured aneurysm. However, despite the strict application of the emergency treatment strategy, the risk of immediate in-hospital rebleeding within 4 hours after admission was not reduced. Thus, more sophisticated and advanced management is required for patients presenting within several hours after SAH. A hybrid operating room with integrated angiographic imaging capabilities would reduce the time from angiography to operation. In addition, antifibrinolytic therapy using EACA could help to reduce the risk of recurrent hemorrhage.

Short-term antifibrinolytic therapy $(<3$ days) with EACA has recently been proposed to reduce the risk of aneurysmal rebleeding for patients undergoing early surgery without increasing the incidence of symptomatic vasospasm and hydrocephalus, as experienced with prolonged antifibrinolytic therapy. $7,18,27$ Thus, patients who receive early treatment for a ruptured aneurysm could benefit from antifibrinolytic therapy. However, the exact role of blood clot lysis in ultra-early rebleeding (within several hours of $\mathrm{SAH}$ ) remains unclear, as even following antifibrinolytic therapy, many cases of rebleeding have been reported to occur on the same day as the initial hemorrhage. ${ }^{7}, 18$

On rare occasions, a recurrent hemorrhage can occur during the induction of general anesthesia and creation of a bone flap for surgical clip placement. In this case, unexpected severe brain swelling can be encountered after dural opening during a craniotomy. The current authors previously reported an intraoperative ultrasonography technique using Paine's point to help with distinguishing a recurrent hemorrhage from acute hydrocephalus and brain edema. ${ }^{25}$

The current study is limited based on a retrospective review of data from a single institution and comparison of data from different time periods. However, the application of propensity score matching to a large case series can reduce the bias due to confounding variables between the two groups. Thus, a reduced risk of rebleeding with resultant clinical improvement was ascertained as a result of the emergency treatment strategy. However, the degree of contribution to improved clinical outcomes for the total patient population cannot be determined due to unevaluated factors, such as clinical experience, the development of endovascular devices, and the application of well-organized multidisciplinary treatment. Therefore, future studies should include a prospective randomized trial comparing the strategy of emergency treatment and urgent treatment combined with antifibrinolytic therapy.

\section{Conclusions}

A strategic change from early treatment to a formal emergency treatment protocol for patients with a ruptured aneurysm significantly reduced the incidence of in-hospital rebleeding, thereby contributing to improved clinical outcomes, especially for patients with good WFNS grades. Therefore, patients with ruptured aneurysms may benefit from a strategy of emergency application of surgical clip placement or endovascular coiling.

\section{References}

1. Ahn JY, Cho JH, Jung JY, Lee BH, Yoon PH: Blister-like aneurysms of the supraclinoid internal carotid artery: chal- 
lenging endovascular treatment with stent-assisted coiling. J Clin Neurosci 15:1058-1061, 2008

2. Birknes JK, Hwang SK, Pandey AS, Cockroft K, Dyer AM, Benitez RP, et al: Feasibility and limitations of endovascular coil embolization of anterior communicating artery aneurysms: morphological considerations. Neurosurgery 59:43-52, 2006

3. Cha KC, Kim JH, Kang HI, Moon BG, Lee SJ, Kim JS: Aneurysmal rebleeding: factors associated with clinical outcome in the rebleeding patients. J Korean Neurosurg Soc 47:119-123, 2010

4. de Gans K, Nieuwkamp DJ, Rinkel GJ, Algra A: Timing of aneurysm surgery in subarachnoid hemorrhage: a systematic review of the literature. Neurosurgery 50:336-342, 2002

5. Fujii Y, Takeuchi S, Sasaki O, Minakawa T, Koike T, Tanaka R: Ultra-early rebleeding in spontaneous subarachnoid hemorrhage. J Neurosurg 84:35-42, 1996

6. Gooderham PA, Steinberg GK: Reflections on the benefits and pitfalls of ultra-early aneurysm treatment after subarachnoid hemorrhage. World Neurosurg 77:261-262, 2012

7. Harrigan MR, Rajneesh KF, Ardelt AA, Fisher WS III: Short-term antifibrinolytic therapy before early aneurysm treatment in subarachnoid hemorrhage: effects on rehemorrhage, cerebral ischemia, and hydrocephalus. Neurosurgery 67:935-940, 2010

8. Ioannidis I, Lalloo S, Corkill R, Kuker W, Byrne JV: Endovascular treatment of very small intracranial aneurysms. Clinical article. J Neurosurg 112:551-556, 2010

9. Kassell NF, Torner JC: Aneurysmal rebleeding: a preliminary report from the Cooperative Aneurysm Study. Neurosurgery 13:479-481, 1983

10. Kassell NF, Torner JC, Jane JA, Haley EC Jr, Adams HP: The International Cooperative Study on the Timing of Aneurysm Surgery. Part 2: Surgical results. J Neurosurg 73:37-47, 1990

11. Kim BM, Park SI, Kim DJ, Kim DI, Suh SH, Kwon TH, et al: Endovascular coil embolization of aneurysms with a branch incorporated into the sac. AJNR Am J Neuroradiol 31:145151,2010

12. Kim JM, Chae YS, Cheong JH, Bak KH, Kim CH, Oh SH: Influence of routine intraoperative ventricular drainage on the incidence of aneurysmal rebleeding. J Korean Neurosurg Soc 36:18-23, 2004

13. Koebbe CJ, Veznedaroglu E, Jabbour P, Rosenwasser RH: Endovascular management of intracranial aneurysms: current experience and future advances. Neurosurgery 59 (5 Suppl 3):S3-93-S3-S102, 2006

14. Kusumi M, Yamada M, Kitahara T, Endo M, Kan S, Iida H, et al: Rerupture of cerebral aneurysms during angiographya retrospective study of 13 patients with subarachnoid hemorrhage. Acta Neurochir (Wien) 147:831-837, 2005

15. Laidlaw JD, Siu KH: Poor-grade aneurysmal subarachnoid hemorrhage: outcome after treatment with urgent surgery. Neurosurgery 53:1275-1282, 2003

16. Laidlaw JD, Siu KH: Ultra-early surgery for aneurysmal subarachnoid hemorrhage: outcomes for a consecutive series of 391 patients not selected by grade or age. J Neurosurg 97:250-258, 2002

17. Lawton MT, Quiñones-Hinojosa A, Chang EF, Yu T: Thrombotic intracranial aneurysms: classification scheme and management strategies in 68 patients. Neurosurgery 56:441-454, 2005
18. Leipzig TJ, Redelman K, Horner TG: Reducing the risk of rebleeding before early aneurysm surgery: a possible role for antifibrinolytic therapy. J Neurosurg 86:220-225, 1997

19. Lubicz B, Lefranc F, Levivier M, Dewitte O, Pirotte B, Brotchi J, et al: Endovascular treatment of intracranial aneurysms with a branch arising from the sac. AJNR Am J Neuroradiol 27:142-147, 2006

20. Moret J, Pierot L, Boulin A, Castaings L, Rey A: Endovascular treatment of anterior communicating artery aneurysms using Guglielmi detachable coils. Neuroradiology 38:800805, 1996

21. Murayama Y, Nien YL, Duckwiler G, Gobin YP, Jahan R, Frazee J, et al: Guglielmi detachable coil embolization of cerebral aneurysms: 11 years' experience. J Neurosurg 98:959-966, 2003

22. Park J: Saccular aneurysm with basal rupture angiographically depicted as an aneurysm with stalk-like narrow neck. Report of 2 cases. J Neurosurg 114:1065-1068, 2011

23. Park J, Kang DH, Chun BY: Superciliary keyhole surgery for unruptured posterior communicating artery aneurysms with oculomotor nerve palsy: maximizing symptomatic resolution and minimizing surgical invasiveness. Clinical article. J Neurosurg 115:700-706, 2011

24. Park J, Woo H, Kang DH, Kim Y, Baik SK: Ruptured intracranial aneurysms with small basal outpouching: incidence of basal rupture and results of surgical and endovascular treatments. Neurosurgery 71:994-1002, 2012

25. Park J, Woo H, Kim GC: Diagnostic usefulness of intraoperative ultrasonography for unexpected severe brain swelling in ultra-early surgery for ruptured intracranial aneurysms. Acta Neurochir (Wien) 154:1869-1875, 2012

26. Park JH, Park IS, Han DH, Kim SH, Oh CW, Kim JE, et al: Endovascular treatment of blood blister-like aneurysms of the internal carotid artery. J Neurosurg 106:812-819, 2007

27. Starke RM, Kim GH, Fernandez A, Komotar RJ, Hickman ZL, Otten ML, et al: Impact of a protocol for acute antifibrinolytic therapy on aneurysm rebleeding after subarachnoid hemorrhage. Stroke 39:2617-2621, 2008

28. Suh SH, Kim BM, Chung TS, Kim DI, Kim DJ, Hong CK, et al: Reconstructive endovascular treatment of intracranial fusiform aneurysms: a 1-stage procedure with stent and balloon. AJNR Am J Neuroradiol 31:155-160, 2010

29. van Rooij WJ, Sluzewski M: Endovascular treatment of large and giant aneurysms. AJNR Am J Neuroradiol 30:12-18, 2009

\section{Author Contributions}

Conception and design: Park. Acquisition of data: Woo. Analysis and interpretation of data: Park, M Kim. Drafting the article: Park. Critically revising the article: all authors. Reviewed submitted version of manuscript: all authors. Approved the final version of the manuscript on behalf of all authors: Park. Statistical analysis: M Kim, Shin, Kwak. Administrative/technical/material support: Kang, YS Kim. Study supervision: Park.

\section{Correspondence}

Jaechan Park, Department of Neurosurgery, Kyungpook National University Hospital, 50, Samduk 2-ga, Jung-gu, Daegu 700-721, Republic of Korea. email: jparkmd@ hotmail.com. 